\section{Positive impact of eculizumab therapy on surgery for Budd- Chiari syndrome in a patient with paroxysmal nocturnal hemoglobinuria and a long- term history of thrombosis}

\author{
Silvia De-la-Iglesia, Hugo Luzardo, \\ Angelina Lemes, Melissa Torres, Maria \\ Teresa Gómez-Casares, Naylen Cruz, \\ Teresa Molero
}

Hematology Department, University Hospital of Gran Canaria Doctor Negrín, Las Palmas de Gran Canaria, Spain

\begin{abstract}
Paroxysmal nocturnal hemoglobinuria (PNH) is associated with severe end-organ damage and a high risk of thrombosis. BuddChiari syndrome, which develops after thrombotic occlusion of major hepatic blood vessels, is relatively common in PNH and has been associated with increased mortality. We report the case of a 46-year-old male with PNH who presented with Budd-Chiari syndrome associated with portal cavernoma, portal hypertension and hypersplenism. In September 2010, the patient suffered gastrointestinal bleeding, hematuria, and elevated plasma lactate dehydrogenase; he started eculizumab therapy with a good response. In October 2012, he developed upper gastrointestinal variceal bleeding and a splenorenal shunt was placed. At the time of writing, the patient remains stable and eculizumab continues to be effective. There is limited data on the use of eculizumab for prevention of hemolysis and its consequences in PNH patients undergoing surgery. Our findings provide evidence for the efficacy and safety of eculizumab in this setting.
\end{abstract}

\section{Introduction}

Paroxysmal nocturnal hemoglobinuria (PNH) is an acquired disease affecting hematopoietic stem cells that is caused by a somatic mutation of the phosphatidylinositol glycan $A$ gene. This mutation affects the synthesis of glucosylphosphatidyl inositol cell membrane anchors, leading to a partial or total deficiency of proteins such as CD55 and CD59 in the surface of all progeny cell populations. ${ }^{1}$ The absence of these regulating proteins results in uncontrolled amplification of the complement system and leaves [ red blood cells (RBCs)], platelets and neutrophils vulnerable to attack, with subsequent hemolysis and platelet activation, resulting in severe endorgan damage and a high risk of thrombosis. Even with the best supportive care, $35 \%$ of patients die within the first ten years after diagnosis. $^{2}$

PNH is considered the most severe acquired thrombophilic state and is associated with a high rate of mortality. ${ }^{3}$ Thrombosis is the main cause of morbidity and mortality and accounts for $40-67 \%$ of related deaths. ${ }^{4-6}$ The risk of thromboembolism alone has been reported as being 62 -fold greater in PNH than in the general population. ${ }^{7}$ Moreover, anticoagulant therapy does not fully protect against thrombosis. ${ }^{5,8,9}$ Thrombotic phenomena in PNH are complex and challenging and can occur even in patients with small PNH clone sizes, minimal hemolysis and no transfusion history. $4,6,10$ Factors associated with thrombosis in $\mathrm{PNH}$ include platelet activation, free hemoglobin toxicity, nitric oxide depletion, absence of other GPI-linked proteins, endothelial dysfunction, and other complement-mediated procoagulation pathways that are independent of haemolysis. ${ }^{6,11}$ Budd-Chiari syndrome is a common complication of PNH and it has been associated with a high risk of mortality among affected patients. ${ }^{6}$ Surgery for Budd-Chiari syndrome has also been associated with high perioperative mortality, with activation of the complement system by anesthesia being one of the main breakthrough hemolysis triggers during surgery. ${ }^{12}$ Surgery in PNH patients is a major challenge due to the increased coagulation risk associated with surgical techniques and with general anesthesia. Since acidosis can cause hemolysis, any state producing hypoxemia, hypoperfusion, hypercapnia, or dehydration must be avoided or properly controlled. ${ }^{12}$ Moreover, even relatively mild infections can trigger hemolysis and activate the complement system. ${ }^{12}$ Additionally, since renal impairment is a frequent complication due to hemosiderin accumulation in proximal tubular epithelial cells, a complete assessment of renal function is recommended before surgery. ${ }^{13}$

Eculizumab, a monoclonal antibody specifically designed to block the complement cascade at the C5 level, is the only approved treatment for PNH. By blocking C5 proteins with high affinity, eculizumab prevents the damage caused both by $\mathrm{C} 5 \mathrm{a}$, a potent anaphylatoxin, and by the membrane attack complex C5b-9, thus preventing the formation of micropores on the surfaces of RBCs, platelets and leucocytes that are responsible for hemolysis and the hyperthrombotic state. It also prevents other life-threatening PNH-related conditions, such as renal impairment. Here we present the case of an adult male patient with a long history of PNH-related thrombosis who successfully underwent surgery for Budd-Chiari syndrome while receiving eculizumab therapy.
Correspondence: Silvia De-la-Iglesia, Hematology Department, University Hospital of Gran Canaria Doctor Negrín, Barranco de la Ballena S/N, 35004 Las Palmas de Gran Canaria, Spain Tel: +34.928.45.00.00.

E-mail: siglini@gobiernodecanarias.org

Key words: Paroxysmal nocturnal haemoglobinuria (PNH); Budd-Chiari syndrome; thrombosis; eculizumab; surgery.

Authors' contributions: SDI, collected the data from the medical record, drafted the manuscript and approved the final version; HL, AL, MT, MTGC, NC and TM, critically reviewed the manuscript and approved the final version.

Conflict of interest: the authors declare no potential conflict of interest.

Acknowledgments: Matthew Reilly, PhD, at InTouch Medical Ltd, provided editorial assistance in the preparation of this manuscript. Dr Reilly's work was funded by Alexion Pharmaceuticals Inc. I would like to record my deep gratitude to Dr. Victor Sánchez Martín, specialist in radiology for his generous assistance and valuable information in providing, supervising and interpreting radiology examinations.

Received for publication: 27 April 2016.

Revision received: 28 June 2016.

Accepted for publication: 30 August 2016.

This work is licensed under a Creative Commons Attribution-NonCommercial 4.0 International License (CC BY-NC 4.0).

(C)Copyright S. De-la-Iglesia et al., 2016

Licensee PAGEPress, Italy

Hematology Reports 2016; 8:6562

doi:10.4081/hr.2016.6562

\section{Case Report}

A 46-year-old male presented at our clinic after having experienced 1-2 hemolytic crises per year since 1985. In January 2001, he suffered a hemolytic crisis associated with thrombosis of the upper longitudinal cerebral sinus, subsequent to which he was diagnosed with PNH based on flow cytometry. He was started on oral anticoagulant therapy with acenocoumarol. In November 2002, following a salmonella infection, he suffered a second crisis with acute renal failure that required dialysis and was resolved in less than a month. Four months later, in March 2003, he presented again with thrombosis, this time affecting the suprahepatic and portal veins (Budd-Chiari syndrome) and associated with portal cavernoma, portal hypertension and hypersplenism. In January 2005, he suffered neutropenia and 
febrile episodes, as well as pneumonia of the right middle lobe.

Between 2003 and 2006, the patient required repeated hospitalizations for several episodes of upper gastrointestinal bleeding caused by esophageal varices. His anticoagulant therapy was stopped in 2005 to reduce the risk of bleeding. Between 2006 and 2010, he suffered asthenia and several episodes of hemoglobinuria accompanied by elevated plasma [lactate dehydrogenase (LDH)]. During this period, his hemoglobin levels remained low (around $8 \mathrm{~g} / \mathrm{dL}$ ), requiring several transfusions. He also had erectile dysfunction.

In September 2010, the patient started treatment with eculizumab at recommended doses, having previously been vaccinated against Neisseria meningitidis. He attained a very good response to eculizumab, with decreases in asthenia, plasma $\mathrm{LDH}$ and bilirubin after the second dose. Overall, eculizumab was well tolerated. Mild cephalalgy was recorded as an adverse event after the first two infusions but was not observed in subsequent examinations. His hemoglobin levels rose after one month of treatment and reached normal levels after one year. In July 2012, the patient was admitted to the emergency room with severe hematemesis and syncope. Gastroscopic examination showed an actively bleeding subcardial varice that was managed with a cyanoacrylate injection. Endoscopic examination also showed grade II fundal varices with risk signs but without active bleeding. His hemoglobin had dropped to $6.8 \mathrm{~g} / \mathrm{dL}$, necessitating the transfusion of two units of RBCs. Three months later, in October 2012, he had another episode of upper gastrointestinal bleeding and it was decided to place a portacaval shunt.

Figure 1 illustrates the pathological condition of the patient's liver at the time or surgery. Numbers inside the figures describe: 1) metal artifact in keeping with embolization material of the gastro-hepatic ligament; 2) diffuse hepatomegaly with irregular and lobulated contours and hypertrophy of the caudate and medial segments of both lobules; 3) chronic thrombosis did not allow the identification of the portal vein. There was ample portal cavernoma with multiple collateral vessels around the common bile duct and cystic duct up to the pancreas head, as well as around the neck and body of the gallbladder, causing dilation of the intrahepatic bile duct. No focal intrahepatic lesions were detected; 4) enlarged spleen (23 $\mathrm{cm}$ of interpolar diameter) with ample collateral vein circulation and splenorenal shunt. No free intraperitoneal fluid was present. Surgery was performed the day after the eculizumab infusion in order to maximize the level of eculizumab in the blood during the procedure. After premedication with midazolam and morphic chloride, and general anesthesia with propofol, remifentanil and cisatracurium, a splenorenal shunt was placed using Warren's technique. The surgical procedure lasted a total of five hours. There was an estimated 500 $\mathrm{mL}$ intraoperative bleeding that required transfusion of one unit of RBCs and one pool of platelets. No renal impairment was observed. The total hematological debit was $1500 \mathrm{~mL}$ due to serohematic draining, but there was no anemia (post-operative hemoglobin $9.9 \mathrm{~g} / \mathrm{dL}$; platelet count $50 \times 10^{9} / \mathrm{L}$ ). The patient did not require hemoderivatives but did receive [lowmolecular-weight heparin (LMWH)] immediately after surgery.

Two days after surgery, the patient showed bleeding through the nasogastric tube, with subsequent anemia (hemoglobin $7.5 \mathrm{~g} / \mathrm{dL}$, platelet count $30 \times 10^{9} / \mathrm{L}$ ) that required the transfusion of two units of RBCs. After the resolution of this episode and for one month thereafter, he received LMWH in order to minimize the risk of thrombosis until he was fully mobilized. LDH levels were monitored regularly to assess the degree of complement blockade

\section{A}

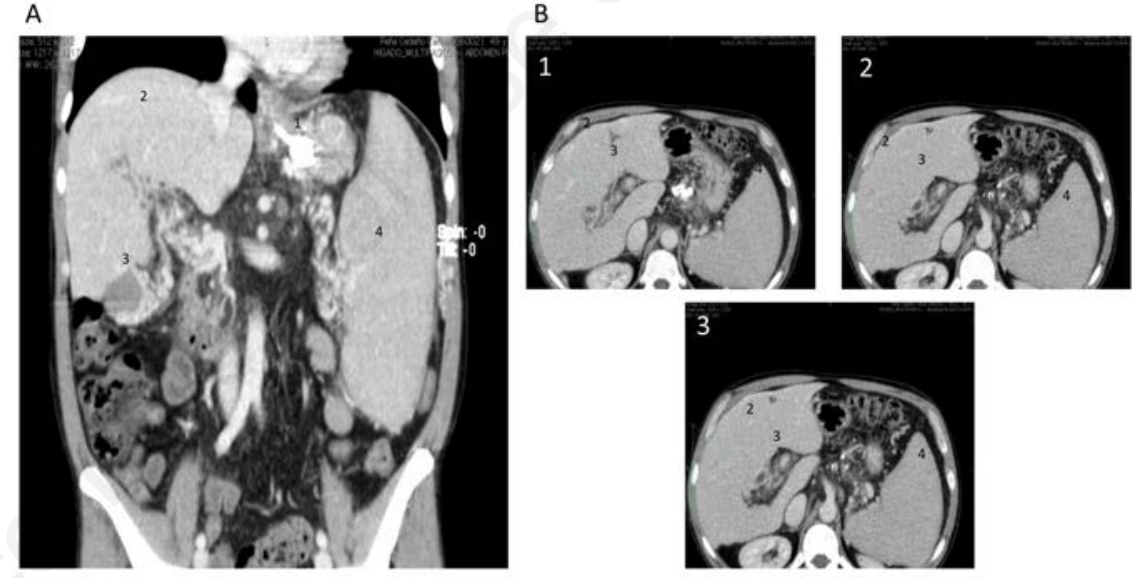

Figure 1. Liver computed tomography at the time of surgery. A) Longitudinal section; B) transversal sections: before (1), at (2) and after portal bifurcation (3).

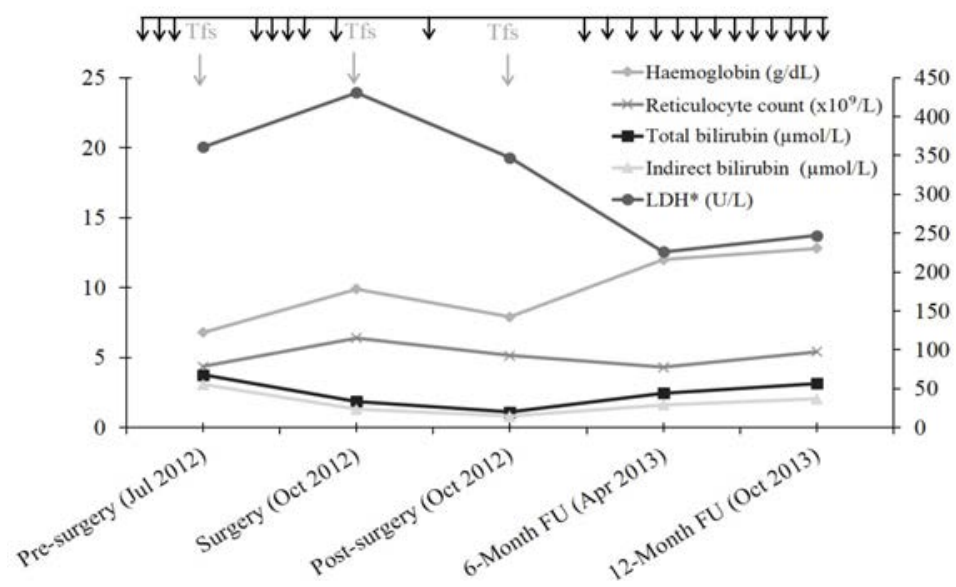

Figure 2. Changes in hematological parameters over time. Individual points represent single measurements in the patient. ${ }^{*} \mathrm{LDH}$ activity $(\mathrm{U} / \mathrm{L})$ shown on right-hand axis; black arrows indicate eculizumab treatment; FU, follow-up; Tfs, transfusion. by eculizumab and there was no need for any dose modification. In October 2013, the patient remained fully recovered, with no further episodes of thromboembolism, hemolysis or gastrointestinal bleeding and no further need for transfusions. The patient continued to receive the standard dose of eculizumab. At the time of writing (September 2015), the patient remains stable, having had only one (J) gastroesophageal variceal bleeding (Jith anemia (hemoglobin 7.5 continues to receive eculizumab every two weeks. Figure 2 illustrates the evolution of the patient's hematological parameters from presurgery (July 2012) to follow-up in October 2013. At further follow-up in May 2015, his count was the following: hemoglobin 9.7 $(10-250)$; total bilirubin $227 \mu \mathrm{mol} /$; indirect bilirubin $1.41 \mu \mathrm{mol} / \mathrm{L}$; reticulocytes $6.65 \%$; corrected reticulocytes $3.78 \%$. The patient is doing sport and his quality of life has 
improved. He has no choluria and tolerates eculizumab treatment well.

\section{Discussion}

To the best of our knowledge, this is the first case report of a successful placement of a splenorenal shunt in a $\mathrm{PNH}$ patient with BuddChiari syndrome and a long history of thrombosis. In fact, there have been few reported cases of surgery on patients receiving eculizumab therapy: three cholecystectomies, ${ }^{14-16}$ one cardiopulmonary bypass,,${ }^{17}$ one splenectomy $^{18}$ and one liver transplant for Budd-Chiari syndrome sequelae. ${ }^{9}$ All these interventions used proper anesthetic and surgical management and attained good postoperative patient outcomes. In contrast with this limited number of published cases of patients treated with eculizumab who require surgery, the association between surgery and complement activation is well described. Complement is activated during surgery as evidenced by a rise in $\mathrm{C} 3 \mathrm{~d} / \mathrm{C} 3$ ratio and increase of $\mathrm{C} 5$ and the terminal/lytic complement compounds, like C5b-9, driving more injury in the tissues and cells (endothelial, erythrocytes, platelets, etc.). A variety of factors can lead to complement activation and hemolysis in patients with PNH undergoing a surgical procedure, such as anesthetic administration, acidosis, inflammation or transfusions. ${ }^{12,14,15,17,19}$ Therefore, patients should be monitored for potential early breakthrough hemolysis after the procedure in order to adjust eculizumab dosage in a timely manner if necessary. Complete C5 blockade by eculizumab during the perioperative period was confirmed in some patients by undetectable terminal complement complex evaluated in vivo after surgery. ${ }^{17} \mathrm{LDH}$ is a very good parameter to estimate the ongoing hemolytic activity. In this regard it is remarkable that in the reported cases of patients treated with eculizumab undergoing surgery LDH levels remained unchanged, indicating that intravascular hemolysis was not significant.

Some specific considerations about the dosage regimen of eculizumab must be taken into account in clinically stable patients with PNH who are being evaluated for elective surgery. Firstly, since the blood concentration of eculizumab (and, consequently, complement blockade) is highest shortly after infusion, it is important to consider scheduling surgery following the administration of the drug in order to maximize its blood levels during the procedure. In the mentioned published cases, eculizumab treatment was administered as close as possible to the day of surgery (maximum 4 days prior to the scheduled date of sur- gery), to minimize any potential exacerbation of intravascular hemolysis. ${ }^{15,17}$ In our patient, eculizumab was administered the day before surgery with good post-operative outcomes Secondly, patients may experience decreased eculizumab concentrations through blood loss during surgery and, therefore, changes in the standard dosage scheme of eculizumab may be considered. In the previous reported cases, eculizumab treatment was administered according to the dosage schedule stated at the prescribing information, which the authors reported to be effective in preventing hemolysis. ${ }^{9,12,14,15,17,18}$ Similarly, in our case there was no need for any dose modification of eculizum$\mathrm{ab}$, and the administration of $900 \mathrm{mg}$ of eculizumab the day before surgery was effective for preventing hemolysis (LDH levels did not increase after the procedure).

\section{Conclusions}

The present case provides a good example of how eculizumab therapy can help to achieve successful surgical intervention for BuddChiari syndrome in at-risk patients with PNH, demonstrating that eculizumab may be useful in the peri-operative period for preventing increased basal hemolysis. Moreover, as confirmed in permeability analysis of the portosystemic shunt by eco-Doppler ultrasound, eculizumab seems to limit posterior or shunt thrombosis. Our findings seem to confirm the suggested efficacy and safety of eculizumab in PNH patients undergoing surgery. Eculizumab prevents hemolysis and its consequences in these patients and thus offers a new perspective on their surgical management.

\section{References}

1. Rosse WF, Ware RE. The molecular basis of paroxysmal nocturnal hemoglobinuria. Blood 1995;86:3277-86.

2. Socié G, Mary JY, de Gramont A, et al., Paroxysmal nocturnal haemoglobinuria: long-term follow-up and prognostic factors. French Society of Haematology. Lancet 1996;348:573-7.

3. Luzzatto L, Risitano AM, Notaro R. Paroxysmal nocturnal hemoglobinuria and eculizumab. Haematologica 2010;95:523-6.

4. Hillmen P, Muus P, Dührsen U, et al., Effect of the complement inhibitor eculizumab on thromboembolism in patients with paroxysmal nocturnal hemoglobinuria. Blood 2007;110:4123-8.

5. Hoekstra J, Leebeek FW, Plessier A, et al., Paroxysmal nocturnal hemoglobinuria in
Budd-Chiari syndrome: findings from a cohort study. J Hepatol 2009;51:696-706.

6. Hill A, Kelly RJ, Hillmen P. Thrombosis in paroxysmal nocturnal hemoglobinuria. Blood 2013;121:4985-96.

7. McKeage K. Eculizumab: a review of its use in paroxysmal nocturnal haemoglobinuria. Drugs 2011;71:2327-45.

8. de Latour RP, Mary JY, Salanoubat C, et al., Paroxysmal nocturnal hemoglobinuria: natural history of disease subcategories. Blood 2008;112:3099-106.

9. Singer AL, Locke JE, Stewart ZA, et al., Successful liver transplantation for BuddChiari syndrome in a patient with paroxysmal nocturnal hemoglobinuria treated with the anti-complement antibody eculizumab. Liver Transpl 2009;15:540-3.

10. Lee JW, Jang JH, Kim JS, et al., Clinical signs and symptoms associated with increased risk for thrombosis in patients with paroxysmal nocturnal hemoglobinuria from a Korean Registry. Int J Hematol 2013;97:749-57.

11. Malato A, Saccullo G, Coco LL, et al., Thrombotic complications in paroxysmal nocturnal haemoglobinuria: a literature review. Blood Transfus 2012;10:428-35.

12. Kathirvel S, Prakash A, Lokesh BN, Sujatha P. The anesthetic management of a patient with paroxysmal nocturnal hemoglobinuria. Anesth Analg 2000;91:1029-31.

13. Jose MD, Lynn KL. Acute renal failure in a patient with paroxysmal nocturnal hemoglobinuria. Clin Nephrol 2001;56:172-4.

14. Ando K, Gotoh A, Yoshizawa S, et al., Successful cholecystectomy in a patient with aplastic anemia-paroxysmal nocturnal hemoglobinuria during eculizumab treatment. Ann Hematol 2012;91:1987-8.

15. Kawano H, Minagawa K, Wakahashi K, et al., Successful management of obstructive jaundice due to gallstones with eculizumab in a patient with paroxysmal nocturnal hemoglobinuria. Intern Med 2012;51:2613-6.

16. Moriyama M, Nagata T, Yoshioka I, et al., A patient with paroxysmal nocturnal hemoglobinuria being treated with eculizumab who underwent laparoscopic cholecystectomy: report of a case. Surg Case Rep 2015;1:57.

17. van Bijnen ST, Vermeer H, Mourisse JM, et al., Cardiopulmonary bypass in a patient with classic paroxysmal nocturnal hemoglobinuria during treatment with eculizumab. Eur J Haematol 2011;87:376-8.

18. Risitano AM, Marando L, Seneca E, Rotoli B. Hemoglobin normalization after splenectomy in a paroxysmal nocturnal hemoglobinuria patient treated by eculizumab. Blood 2008;112:449-51.

19. Rosse WF, Nishimura J. Clinical manifestations of paroxysmal nocturnal hemoglobinuria: present state and future problems. Int $\mathrm{J}$ Hematol 2003;77:113-20. 